\title{
萗二酰亚胺手性衍生物的合成及其场效应晶体管性能研究
}

\author{
周 敏 $a, b$ 李 晶 $b$ 程 杰 $b$ 葛从伍 ${ }^{*}, b$ 程探宇*,a 高希珂*,b \\ ( ${ }^{a}$ 上海师范大学化学与材料科学学院 上海 200234) \\ ( $b$ 中国科学院上海有机化学研究所 中国科学院有机功能分子合成与组装化学重点实验室 上海 200032)
}

\begin{abstract}
摘要 䒺二酰亚胺类共轭分子是一类经典且被广泛研究的有机半导体材料. 在本工作中, 通过亲芳核取代反应将手性 环已二胺引入荎二酰亚胺的两端合成了光学纯化合物(I-R, I-S ), 内消旋化合物 $(\mathrm{I}-\boldsymbol{R}, \boldsymbol{S})$, 以及经 I- $\boldsymbol{R}$ 和 I-S 等物质的量共 混得到的外消旋混合物(I-rac), 并对这些化合物的紫外-可见吸收光谱、圆二色谱及电化学性质进行了研究. 光学纯 $(\mathbf{I}-\boldsymbol{R}, \mathbf{I}-\boldsymbol{S})$ 和消旋体 $(\mathbf{I}-\boldsymbol{R}, \boldsymbol{S}, \mathbf{I}-\mathbf{r} \boldsymbol{a c})$ 材料在溶液状态下的光谱吸收完全一致，但其薄膜吸收存在差异，消旋体薄膜的最大吸 收峰较光学纯材料红移了约 $5 \mathrm{~nm}$, 这表明它们的聚集态结构有所不同. 以这些化合物或混合物为活性层制备的有机场 效应晶体管(OFET)在氮气条件下均表现出 $\mathrm{p}$ 型半导体特征. 其中, 内消旋体(I-R, S 和外消旋体(I-rac) OFET 器件的空穴 迁移率分别为 0.074 和 $0.075 \mathrm{~cm}^{2} \cdot \mathrm{V}^{-1} \cdot \mathrm{s}^{-1}$, 均高于光学纯材料 $\mathbf{I}-\boldsymbol{R}$ 和 $\mathbf{I}-\boldsymbol{S}$ 的器件性能 (空穴迁移率约为 $0.04 \mathrm{~cm}^{2} \cdot \mathrm{V}^{-1} \cdot \mathrm{s}^{-1}$ ). 研究结果表明, 手性诱导分子组装产生不同的聚集态结构, 从而对材料的器件性能产生一定影响, 这为荎二酰亚胺类 有机功能分子的发展提供了新思路.
\end{abstract}

关键词＼cjkstart䒺二酰亚胺; 手性; 聚集态结构; 有机场效应晶体管

\section{Synthesis and Field-Effect Characteristics of the Chiral Naphthalene Diimide Derivatives}

\author{
Zhou, Min ${ }^{a, b} \quad$ Li, Jing ${ }^{b} \quad$ Cheng, Jie $^{b} \quad$ Ge, Congwu* ${ }^{*, b} \quad$ Cheng, Tanyu*,a $\quad$ Gao, Xike*,b \\ ( ${ }^{a}$ College of Chemistry and Materials Science, Shanghai Normal University, Shanghai 200234) \\ ( ${ }^{b}$ Key Laboratory of Synthetic and Self-Assembly Chemistry for Organic Functional Molecules, Shanghai Institute of \\ Organic Chemistry, Chinese Academy of Science, Shanghai 200032)
}

\begin{abstract}
Naphthalene diimides (NDI)-based conjugated molecules were a class of typical and widely studied organic semiconducting materials. The optically pure compounds (I-R and $\mathbf{I}-\boldsymbol{S})$ and mesomer $(\mathbf{I}-\boldsymbol{R}, \boldsymbol{S})$ by $\mathrm{S}_{\mathrm{N}} \mathrm{Ar}$ reactions between tetraboromo-NDI and 1,2-diaminocyclohexane were designed and synthesized, moreover, the racemic mixture (I-rac) was obtained by mixing $\mathbf{I}-\boldsymbol{R}$ and $\mathbf{I}-\boldsymbol{S}$ equimolarly. The UV-vis absorption spectroscopy, cyclic voltammetry, and circular dichroism of four materials were studied. The UV-Vis absorptions in solution were consistent, while the absorptions in film were slightly different between the optically pure compounds and the mesomer or raceme, indicating the different aggregate structures. Accordingly, the organic field-effect transistors (OFET) by using these materials as the active layer were prepared. They all exhibited p-type organic semiconducting characteristics under nitrogen conditions. Meanwhile, mesomer (I-R,S) and raceme (I-rac) showed better OFET device performance (hole mobility: about $0.075 \mathrm{~cm}^{2} \cdot \mathrm{V}^{-1} \cdot \mathrm{s}^{-1}$ ) than that of the optically pure compounds I- $\boldsymbol{R}$ and $\mathbf{I}-\boldsymbol{S}$ (hole mobility: about $0.04 \mathrm{~cm}^{2} \cdot \mathrm{V}^{-1} \cdot \mathrm{s}^{-1}$ ). The atomic force microscopy (AFM) studies demonstrated that thin films of mesomer (I-R,S) and raceme (I-rac) annealed at $100{ }^{\circ} \mathrm{C}$ showed larger form size of microstructures with fewer grain boundaries, which was consistent with the enhanced device performance after thermal annealing. This paper proved that the chirality influenced the molecule self-assembly, packing structure and the resulting device performance.

Keywords naphthalene diimides; chirality; aggregate structure; organic field-effect transistors
\end{abstract}

如果一个物体不能与其镜像重合，则该物体具有手 性. 手性在自然界中普遍存在，一直是化学、材料科学、
生物、医学和物理等多学科领域的核心研究课题之 - ${ }^{[1-3]}$. 自 1848 年巴斯德发现分子的旋光性 ${ }^{[4]}$ 以来，人们

\footnotetext{
* Corresponding authors. E-mail: gecongwu@sioc.ac.cn; tycheng@shnu.edu.cn; gaoxk@mail.sioc.ac.cn Received May 12, 2021; revised July 15, 2021; published online August 17, 2021.

Project supported by the National Natural Science Foundation of China (Nos. 21522209, 21790362), and the Science and Technology Commission of Shanghai Municipality (Nos. 19XD1424700, 18JC1410600)

国家自然科学基金(Nos. 21522209, 21790362)、上海市科学技术委员会(Nos. 19XD1424700, 18JC1410600)资助项目.
} 
对手性的研究不断深入. 手性有机材料在电子器件逐渐 显示出应用潜力. 例如, Reinitzer ${ }^{[5]}$ 第一个发现热致液晶 的中间相是一种基于手性胆固醇的有机分子, 开启了液 晶研究领域. Chen 等 ${ }^{6]}$ 报道了一类带有手性侧链的聚合 物, 发光不对称因子高达 0.35 . Fucher等 ${ }^{[7]}$ 将传统的非手 性聚合物和手性芳香分子进行掺杂, 掺杂后的聚合物具 有了圆偏振发光(CPL)的性质. Tang 等 ${ }^{[8]}$ 合成了一系列 具有聚集诱导发光(AIE)性质的圆偏振发光材料, 有趣 的是这类化合物在溶液状态下没有圆二色性和 CPL 性 质, 但在薄膜态或聚集态下却出现了手性信号, 表明手 性诱导分子产生不同的聚集态结构. Nakamura 等[9]报道 了一种螺手性分子, 其光学纯材料表现为 $\mathrm{n}$ 型半导体电 荷传输性质, 而外消旋体表现为 $\mathrm{p}$ 型, 说明手性影响了 分子堆积, 进而使材料表现出不同的电荷传输性质. 人 们普遍认为相同构型分子之间的相互作用优于相反构 型分子之间的相互作用, 即分子倾向于同手性组装. 然 而越来越多的研究发现, 消旋体较同手性组装的光学纯 材料表现出更优的性能. Nguyen 等 ${ }^{[10]}$ 报道了三种 DPP 类有机共轭小分子手性材料, 其中内消旋体材料具有更 好的平面性和更优的电荷传输性能. Fucher 等 ${ }^{[11]}$ 发展了 一系列氮杂螺烯类有机分子, 外消旋体的空穴迁移率较 同手性组装的光学纯材料增加了 80 多倍. 2019 年, 本课 题组 ${ }^{[12]}$ 报道了一类轴手性荎二酰亚胺类小分子材料, 外消旋体材料的电子迁移率较光学纯材料高出两个数 量级. 这些研究结果表明, 手性对分子组装/聚集态结构 以及材料的电荷传输性能有显著影响, 相关研究能够实 现对分子聚集态和材料器件性能的调控.

䒺二酰亚胺(NDI)衍生物是一类经典且被广泛研究 的有机半导体材料, 具有较低的 LUMO 能级、较高的热 稳定性和化学稳定性, 常用作为 $\mathrm{n}$ 型有机半导体材料的 结构砌块 ${ }^{[13-16]}$. 本课题组与合作者 ${ }^{[17]}$ 报道了一类 $\pi$-拓展 的 NDI 类衍生物 NDI-DTYM2, 其 OFET 器件在空气中 测得电子迁移率高达 $3.5 \mathrm{~cm}^{2} \cdot \mathrm{V}^{-1} \bullet \mathrm{s}^{-1}$, 是目前已报道的 综合性能最好的 $\mathrm{n}$ 型有机小分子之一. 随后, 本课题组 发展了共轭骨架 NDI-DTYA2, 释放出两个反应位点, 进一步扩大了分子的可设计性 ${ }^{[18]} .2018$ 年, 我们向 NDI-DTYA2 的分子骨架引入了不同的双环(杂)芳烃, 所得材料的 OFET 器件电子迁移率最大为 $0.37 \mathrm{~cm}^{2}$. $\mathrm{V}^{-1} \cdot \mathrm{s}^{-1}$, 同时材料的吸收光谱延伸至 $920 \mathrm{~nm}$, 可用作近 红外材料 ${ }^{[19]}$. 近期, 我们报道了含硒 $\mathrm{n}$-型有机半导体材 料 NDI-DSYM2, 材料薄膜不经退火处理电子迁移率即 可高达 $1.0 \mathrm{~cm}^{2} \cdot \mathrm{V}^{-1} \cdot \mathrm{s}^{-1[20]}$. 这些研究结果表明, 对 NDI 共轭骨架进行修饰和拓展是一项有意义的研究工作. 基 于此, 我们将手性环己二胺引入 NDI 分子骨架并研究手 性对这类分子聚集态结构和电荷传输性能的影响.

\section{1 结果与讨论}

\section{1 合成与表征}

目标化合物 I- $\boldsymbol{R}, \mathbf{I}-\boldsymbol{S}$ 和 I- $\boldsymbol{R}, \boldsymbol{S}$ 的合成路线如 Scheme 1 所示. 从起始原料 1,4,5,8-䒬四酸酐出发, 经两步反 应 ${ }^{[21-22]}$ 得到中间产物 I-3, 最后 I-3 和手性环已二胺发生 亲芳核取代反应得到光学纯化合物 I-R (64\%), I-S (56\%) 以及内消旋体 I-R,S (68\%). 随后, 将等量的 I- $\boldsymbol{R}$ 和 I-S 溶于二氯甲烷, 经甲醇沉析得到了外消旋体 I-rac.

用 ${ }^{1} \mathrm{H} N M R 、{ }^{13} \mathrm{C} N M R$ 、高分辨质谱、红外光谱及 元素分析对目标化合物 I-R, I-S 和 I-R,S 的化学结构进 行了表征. 在室温条件下, 化合物 I-R, I-S 和 I- $R, S$ 在二 氯甲烷、氯仿和甲苯等有机溶剂中均具有较好的溶解性, 因此可用溶液法制备其半导体器件. 热重分析(TGA)曲 线显示光学纯材料(I-R, I-S 和消旋体材料(I-R,S, I-rac) 失重 $5 \%$ 时的温度分别为 $416,416,418$ 和 $424{ }^{\circ} \mathrm{C}$ (表 1 ), 表明四者均具有良好的热稳定性，消旋体材料的热稳定 性稍好. 差示扫描量热(DSC)曲线显示四者均存在一对 明显的吸放热峰, 分别对应于它们的熔点和结晶点, 表 明四者均具有可逆的相变过程. 同时, 光学纯材料 $\mathbf{I}-\boldsymbol{R}$ 和 I-S 表现出一致的吸放热行为, 均在 $85{ }^{\circ} \mathrm{C}$ 存在一个固 固相变的吸热峰, 内消旋体 I-R,S 的固固相变吸热峰出 现在 $178{ }^{\circ} \mathrm{C}$, 而外消旋体 I-rac 未观察到这样的固固相 变. 光学纯材料(I-R, I-S 和消旋体材料(I-R,S, I-rac) 不 同的热力学行为表明它们的分子聚集态结构有所不同.

表 $1 \mathrm{I}-\boldsymbol{R}, \mathrm{I}-\mathrm{S}, \mathrm{I}-\mathrm{R}, \mathrm{S}$ 和 I-rac 的光谱和热力学数据 Table 1 Optical and electrochemical data for I- $\boldsymbol{R}, \mathbf{I}-\boldsymbol{S}, \mathbf{I}-\boldsymbol{R}, \boldsymbol{S}$ and I-rac

\begin{tabular}{|c|c|c|c|c|c|}
\hline \multirow{2}{*}{ Compd. } & \multicolumn{2}{|c|}{$\lambda_{\max } / \mathrm{nm}$} & \multirow{2}{*}{$E_{\mathrm{g}}{ }^{a} / \mathrm{eV}$} & \multirow{2}{*}{$T_{\mathrm{d}}^{b} /{ }^{\circ} \mathrm{C}$} & \multirow{2}{*}{$\begin{array}{c}T_{\mathrm{pt}}{ }^{c} /{ }^{\circ} \mathrm{C} \\
\text { Heating/Cooling }\end{array}$} \\
\hline & Solid & Film & & & \\
\hline I-R & 630 & 639 & 1.78 & 416 & $277 / 283$ \\
\hline $\mathrm{I}-\mathrm{S}$ & 630 & 639 & 1.78 & 416 & $277 / 283$ \\
\hline $\mathrm{I}-\boldsymbol{R}, \boldsymbol{S}$ & 630 & 644 & 1.78 & 418 & $277 / 287$ \\
\hline I-rac & 630 & 644 & 1.78 & 424 & $285 / 292$ \\
\hline
\end{tabular}

${ }^{a}$ Calculated from absorption onset of film using equation: $E_{\mathrm{g}}=1240 / \lambda_{\text {onset }} \mathrm{eV}$.

${ }^{b}$ Measured by TGA under a nitrogen flow at a temperature ramp of $10^{\circ} \mathrm{C} / \mathrm{min}$. ${ }^{c}$ Obtained from DSC scans under nitrogen at a scan rate of $10^{\circ} \mathrm{C} / \mathrm{min}$.

\section{2 单晶结构}

为了研究分子及分子聚集态结构, 通过溶液扩散法 得到了 I-R 和 I-S 的单晶(未得到 I-R,S 的单晶), 并对其 进行了结构解析. 单晶结构解析表明, 化合物 I-R 和 I-S 的单晶都属于三斜晶系, 具有扭曲的共轭骨架. 其中化 合物 $\boldsymbol{I}-\boldsymbol{R}$ 的晶胞参数分别为 $a=0.51627 \mathrm{~nm}, b=1.1369$ $\mathrm{nm}, c=1.6632 \mathrm{~nm} ; \alpha=98.765^{\circ}, \beta=95.470^{\circ}, \gamma=101.852^{\circ}$. 两个 $N$-烷基链以及同侧的两个氮原子都分别位于 NDI 平面的上下方, 呈现出椅式的分子构型, 同侧两个上下 的氮原子又分别与环已二胺的碳原子键连接形成了两 
<smiles>O=C1OC(=O)c2ccc3c4c(ccc1c24)C(=O)OC3=O</smiles>

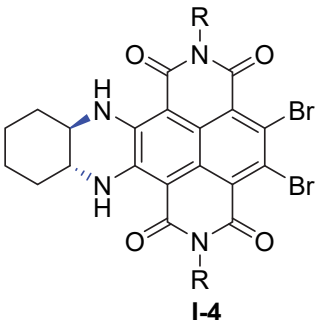<smiles>C[C@@H]1CCCC[C@H]1N</smiles><smiles>O=C1OC(=O)c2c(Br)c(Br)c3c4c(c(Br)c(Br)c(c24)C1=O)C(=O)OC3=O</smiles>

$1-2$
1) $\mathrm{RNH}_{2}, \mathrm{CH}_{2} \mathrm{Cl}_{2}$, reflux $46 \%$

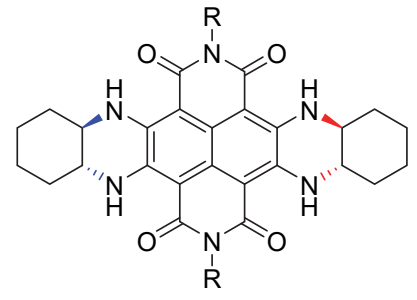<smiles>[R]N1C(=O)c2c(Br)c(Br)c3c4c(c(Br)c(Br)c(c24)C1=O)C(=O)N([R])C3=O</smiles>

I-3<smiles>[R]N1C(=O)c2c(Br)c(Br)c3c4c(c(Br)c(Br)c(c24)C1=O)C(=O)N([R])C3=O</smiles>

$\mathrm{I}-\mathrm{R}, \mathrm{S}$

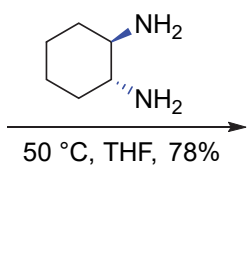

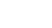

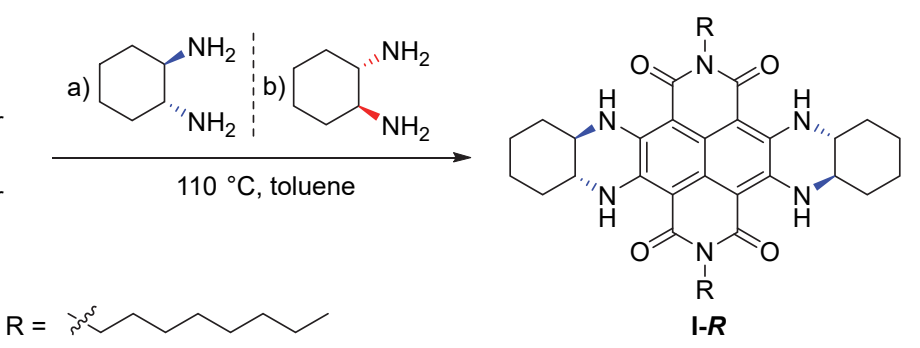

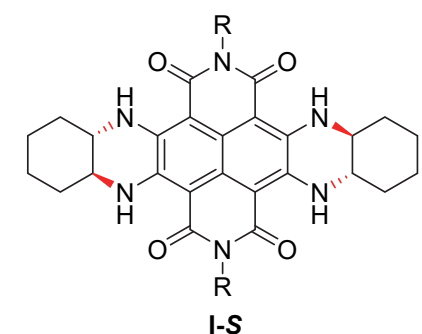

图式 1 化合物 I- $\boldsymbol{R}, \mathbf{I}-\boldsymbol{S}$ 和 I-R,S 的合成路线

Scheme 1 Synthetic routes of compounds I- $R$, I-S and I-R,S

个平面, 经模拟两平面依次与 NDI 所在平面形成的二面 角分别为 $15.29^{\circ}$ 和 $17.10^{\circ}$. 此外, 如图 1 所示, 分子沿着 $b$ 轴方向形成一维错位的 $\pi-\pi$ 堆积, I- $\boldsymbol{R}$ 相邻分子间最近 的 $\pi-\pi$ 堆叠距离约为 $0.3135 \mathrm{~nm}$. 相邻分子间具有较大的 $\pi-\pi$ 重叠面积, 分子形成平行的一维柱状紧密堆积. 化 合物 I-S 的单晶结构与化合物 I- $\boldsymbol{R}$ 类似.

\section{3 光谱和电化学}

用紫外-可见吸收光谱对光学纯材料 $(\mathbf{I}-\boldsymbol{R}, \mathbf{I}-\boldsymbol{S})$ 和消 旋体材料(I-R,S, I-rac $)$ 在二氯甲烷溶液以及薄膜状态下 (图 2)的光学性质进行了研究. 它们在二氯甲烷溶液中 表现出完全相同的光谱吸收行为, 最大吸收峰在 630 $\mathrm{nm}$, 其中 500 700 $\mathrm{nm}$ 的吸收带归因于分子内电荷转移 (ICT). 从溶液到薄膜, I-R, I-S, I-R,S 和 I-rac 的吸收带 均发生红移, 这表明在固态下四种材料都形成了 $J$ 型堆 积 ${ }^{[23]}$. 此外, 消旋体(I-R,S, I-rac) 薄膜的最大吸收峰较 光学纯(I-R, I-S) 红移了约 $5 \mathrm{~nm}$. 四种材料在薄膜状态下 紫外-可见吸收光谱的差异进一步表明光学纯 $(\mathbf{I}-\boldsymbol{R}, \mathbf{I}-\boldsymbol{S})$ 和消旋体(I-R,S, I-rac) 材料的聚集态结构存在一定差别, 这与差示扫描量热(DSC)结果相一致.

圆二色谱(ECD)被用来评价材料对圆偏振光的响应 性质, 我们表征了材料在溶液状态下对左、右偏振光的 响应情况. 化合物的 ECD 谱与其发色团的吸收光谱密
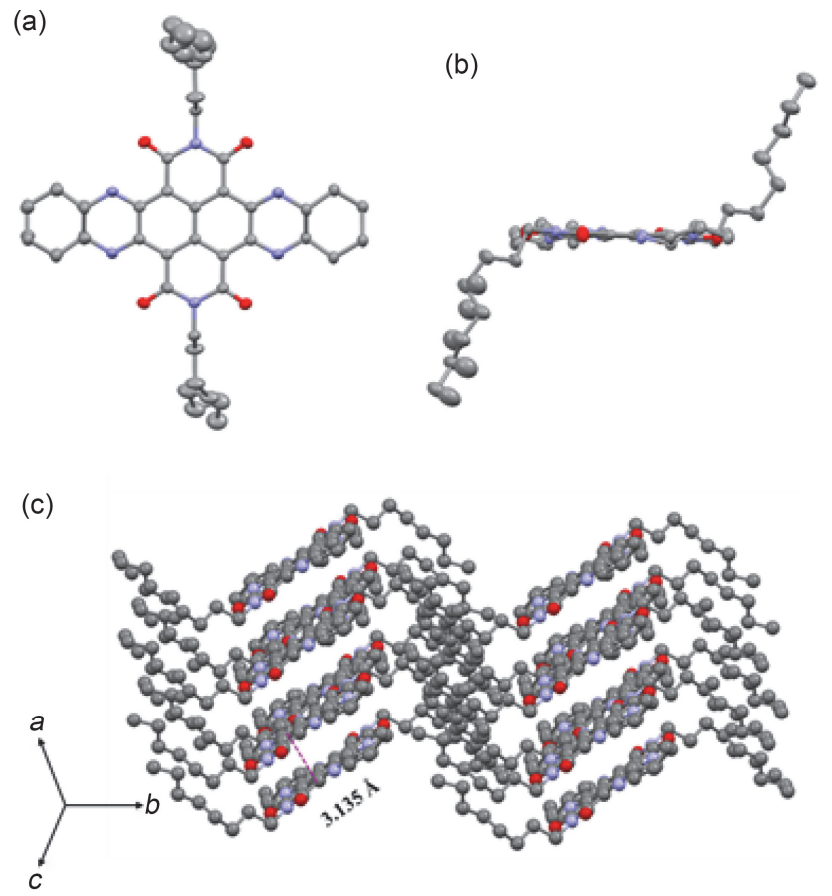

图 1 I-R 的单晶结构图

Figure 1 Crystal structure of I- $\boldsymbol{R}$ (a) Top view, (b) side view, and (c) molecular packing

切相关, 因此在二氯甲烷溶液中, I-R 和 I-S 的紫外区与 

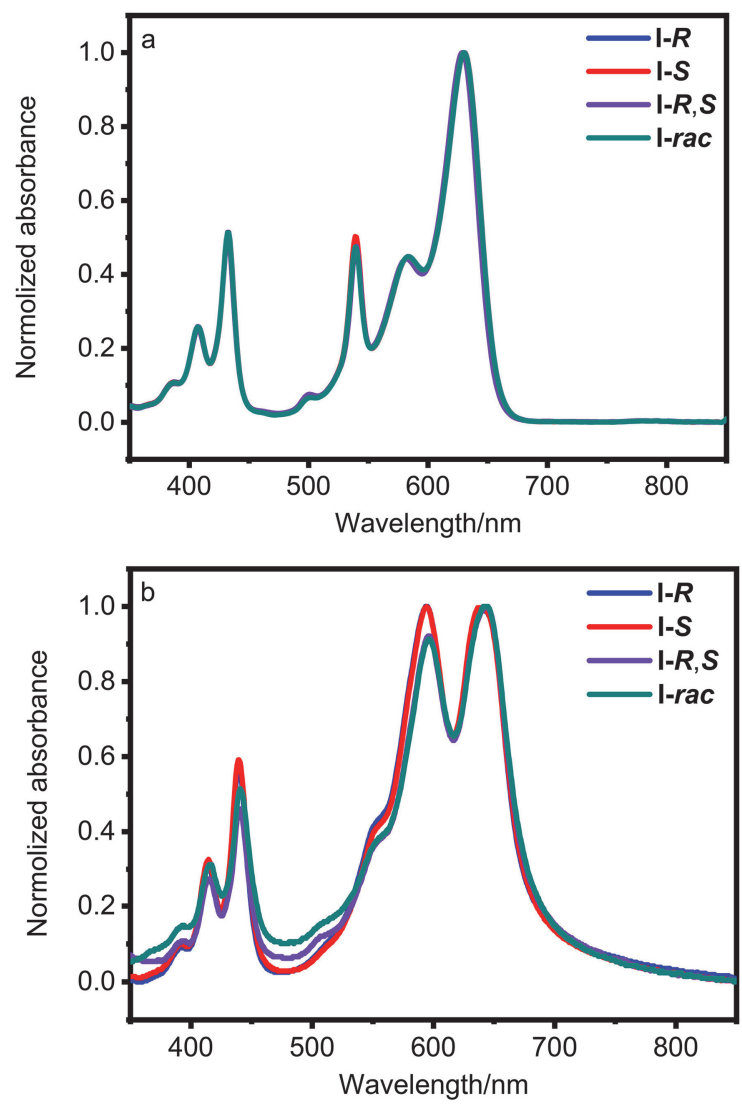

图 $2 \mathrm{I}-\boldsymbol{R}, \mathrm{I}-\mathrm{S}, \mathrm{I}-\mathrm{R}, \mathrm{S}$ 和 I-rac 的紫外-可见吸收光谱

Figure 2 UV-Vis absorption spectra of I-R, I-S, I- $R, S$ and I-rac (a) In dichloromethane solution, (b) as-spun thin films

可见区强烈的镜像 Cotton 效应(图 3a)表明, 环己基的手 性中心使发色团 NDI 处于不对称的手性环境中, 且 CD 信号的峰位置(500 700 nm) 和其紫外-可见光谱的最大 吸收波长 $(630 \mathrm{~nm})$ 相一致.

采用循环伏安法 $(\mathrm{CV})$ 测试了光学纯材料 $(\mathbf{I}-\boldsymbol{R}, \mathbf{I}-\boldsymbol{S})$ 和消旋体材料(I-R,S, I-rac) 的电化学性质. 如图 $3 b$ 所示, 它们的二氯甲烷溶液在正电位表现出两对一致的可逆 氧化峰, 计算出其第一半波氧化电位约为 $0.35 \mathrm{~V}$. 在相 同条件下，用二茂铁作外标并设定其真空能级为 -4.80 $\mathrm{eV}$, 测得二茂铁相对甘录电极的电位为 $0.47 \mathrm{~V}$, 由公式 $E_{\mathrm{HOMO}}=-4.33 \mathrm{eV}-E_{\mathrm{oxl}}^{\text {onset }}$ (经二茂铁校正) 计算出 HOMO 为 $-4.68 \mathrm{eV}$, 从溶液吸收光谱中的末端吸收估 算出其光学带隙为 $1.78 \mathrm{eV}$, 由公式 $E_{\mathrm{LUMO}}=E_{\mathrm{HOMO}}+E_{\mathrm{g}}$ 计算出 LUMO 为 $-2.90 \mathrm{eV}$.

\section{4 场效应晶体管性能}

为了研究手性对聚集态结构和电荷传输性能的影 响, 制备了基于光学纯(I- $R, I-S)$ 和消旋体(I-R,S, I-rac) 材料的底柱极顶接触(BGTC)的薄膜 OFET 器件, 并在 氮气中测试了其场效应性能, 数据列于表 2. 如图 4 所 示, 四种材料均表现出典型的 $\mathrm{p}$ 型电荷传输性质. 四者
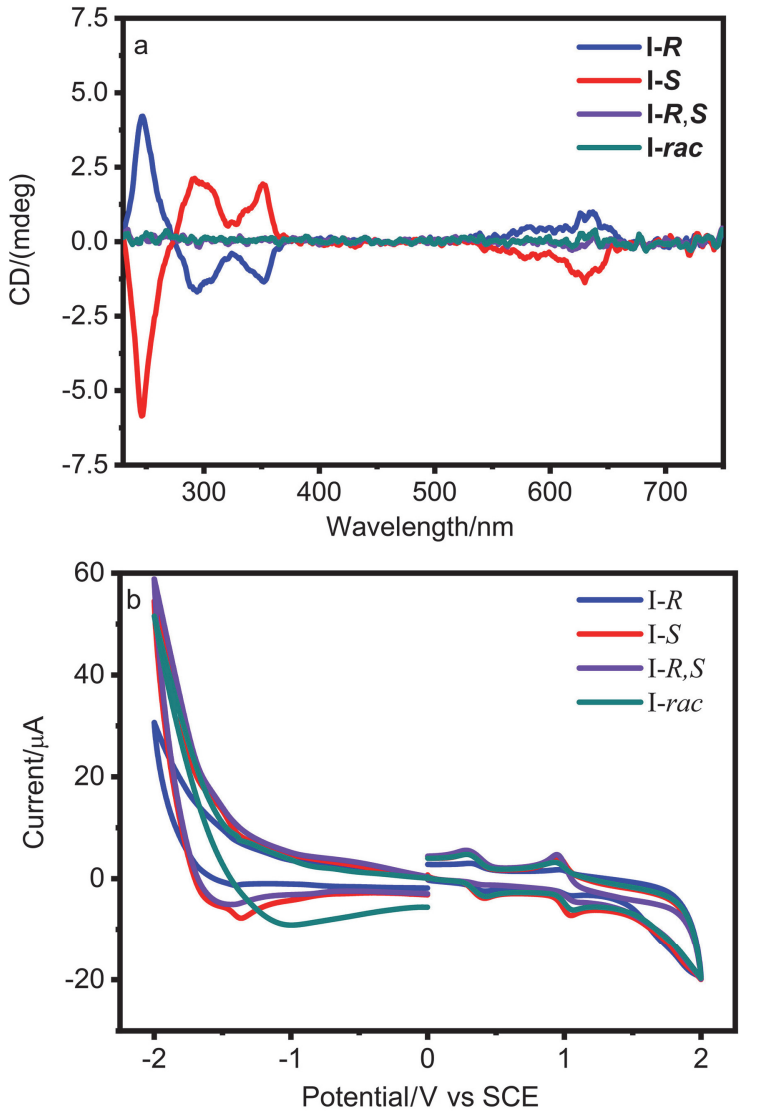

图 $3 \mathrm{I}-\boldsymbol{R}, \mathrm{I}-\mathrm{S}, \mathrm{I}-\boldsymbol{R}, S$ 和 I-rac 在二氯甲烷溶液中的(a)圆偏振吸 收光谱和(b)循环伏安曲线

Figure 3 (a) CD spectra and (b) cyclic voltammogram of I-R, $\mathbf{I}-\boldsymbol{S}, \mathbf{I}-\boldsymbol{R}, \boldsymbol{S}$ and $\mathbf{I}-\boldsymbol{r a c}$ in dichloromethane solution

$0.1 \mathrm{~mol} / \mathrm{L} \mathrm{Bu}_{4}^{+} \mathrm{NPF}_{6}^{-}$as the electrolyte, $\mathrm{SCE}$ as reference electrode, scan rate of $100 \mathrm{mV} / \mathrm{s}$

均在 $100{ }^{\circ} \mathrm{C}$ 热退火的薄膜器件中表现出最优的性能, 内 消旋体 $(\mathbf{I}-\boldsymbol{R}, \boldsymbol{S})$ 和外消旋体(I-rac) 的 OFET 空穴迁移率分 别为 0.074 和 $0.075 \mathrm{~cm}^{2} \cdot \mathrm{V}^{-1} \cdot \mathrm{s}^{-1}$, 均高于光学纯材料 $\mathbf{I}-\boldsymbol{R}$ 和 I-S (约为 $0.04 \mathrm{~cm}^{2} \cdot \mathrm{V}^{-1} \cdot \mathrm{s}^{-1}$ ). 同时, 随着 $180{ }^{\circ} \mathrm{C}$ 条件 下的进一步热退火, 基于消旋体材料薄膜的 OFET 器件 性能仅略有下降, 而相同条件下, 光学纯材料(I-R, I-S) 薄膜 OFET 器件的空穴迁移率均下降至 $4.6 \times 10^{-3} \mathrm{~cm}^{2}$. $\mathrm{V}^{-1} \cdot \mathrm{s}^{-1}$. 此外, 在 $180{ }^{\circ} \mathrm{C}$ 热退火后, 光学纯 $(\mathbf{I}-\boldsymbol{R}, \mathbf{I}-\boldsymbol{S})$ OFET 器件的开关比也比消旋体(I-R,S, I-rac)材料低了 一个数量级, 研究结果表明: 消旋体薄膜的聚集态结构 较光学纯材料具有更优的电荷传输性能. 如表 2 所示, 四个化合物退火后其 OFET 器件的阈值电压比退火前升 高了一个数量级. 鉴于阈值电压的大小主要受有机半导 体层与绝缘层间界面的电荷陷阱密度、源漏电极接触质 量等因素的影响. 推测, 退火前后有机半导体层薄膜形 貌/微观结构发生了较大改变, 引起金属电极和有机半 导体层的接触界面、有机半导体层与绝缘层间接触界面 

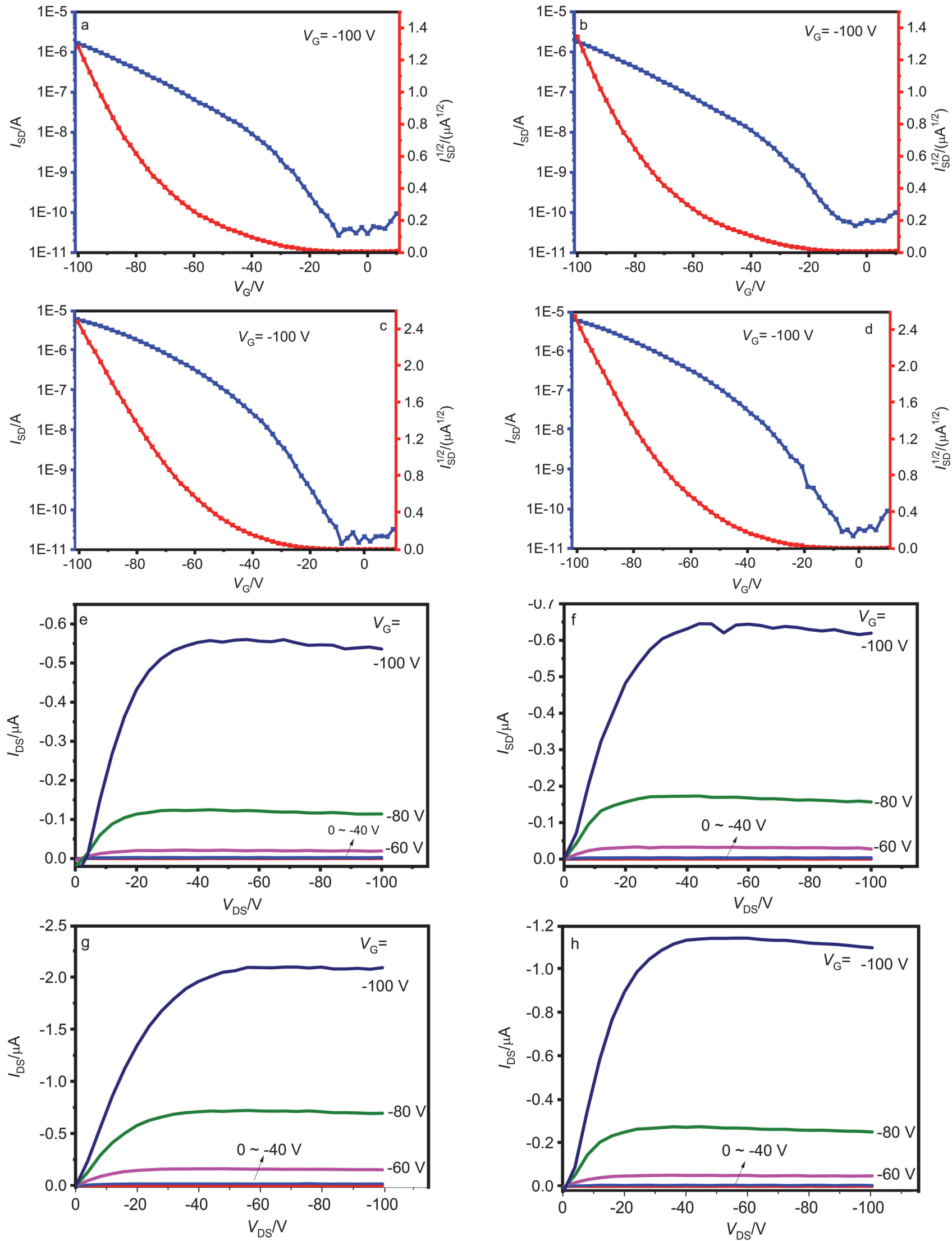

图 4 基于材料 $\mathbf{I}-\boldsymbol{R}(\mathrm{a}, \mathrm{e}), \mathbf{I}-\boldsymbol{S}(\mathrm{b}, \mathrm{f}), \mathbf{I}-\boldsymbol{R}, \boldsymbol{S}(\mathrm{c}, \mathrm{g})$ 和 $\mathbf{I}-\boldsymbol{r a c}(\mathrm{d}, \mathrm{h})$ 的 OFET 器件在 $100{ }^{\circ} \mathrm{C}$ 退火条件下的转移和输出曲线

Figure 4 Typical transfer and output curves of OFET devices based on I- $\boldsymbol{R}(\mathrm{a}, \mathrm{e}), \mathbf{I}-\boldsymbol{S}$ (b, f), I- $\boldsymbol{R}, \boldsymbol{S}$ (c, g) and I-rac (d, h) annealed at $100{ }^{\circ} \mathrm{C}$ 
表 2 I- $R$, I-S, I-R,S 和 I-rac 的 OFET 器件性能

Table 2 Characteristics of OFET devices based on I- $\boldsymbol{R}, \mathbf{I}-\boldsymbol{S}$, $\mathbf{I}-\boldsymbol{R}, \boldsymbol{S}$ and I-rac

\begin{tabular}{lccrc}
\hline Compd. & $\begin{array}{c}\text { Annealing } \\
\text { temperature } /{ }^{\circ} \mathrm{C}\end{array}$ & $\begin{array}{c}\mu_{\mathrm{h}, \max } / \\
\left(\mathrm{cm}^{2} \cdot \mathrm{V}^{-1} \cdot \mathrm{s}^{-1}\right)\end{array}$ & $V_{\mathrm{T}} / \mathrm{V}$ & $I_{\text {On }} / I_{\text {off }}$ \\
\hline \multirow{4}{*}{$\boldsymbol{I} \boldsymbol{R}$} & As-spun & $3.2 \times 10^{-5}$ & 6.1 & $10^{2} \sim 10^{3}$ \\
& 100 & $4.0 \times 10^{-2}$ & -62.9 & $10^{4} \sim 10^{5}$ \\
& 180 & $4.6 \times 10^{-3}$ & -40.4 & $10^{3} \sim 10^{4}$ \\
$\mathbf{I} \boldsymbol{S}$ & As-spun & $3.8 \times 10^{-5}$ & 1.8 & $10^{2} \sim 10^{3}$ \\
& 100 & $3.6 \times 10^{-2}$ & -62.4 & $10^{4} \sim 10^{5}$ \\
& 180 & $4.6 \times 10^{-3}$ & -42.6 & $10^{3} \sim 10^{4}$ \\
$\mathbf{I}-\boldsymbol{R}, \boldsymbol{S}$ & As-spun & $4.9 \times 10^{-5}$ & 2.9 & $10^{2} \sim 10^{3}$ \\
& 100 & $7.4 \times 10^{-2}$ & -50.6 & $10^{4} \sim 10^{5}$ \\
& 180 & $1.4 \times 10^{-2}$ & -40.0 & $10^{4} \sim 10^{5}$ \\
$\mathbf{I}-\boldsymbol{r a c}$ & As-spun & $1.7 \times 10^{-5}$ & 3.0 & $10^{2} \sim 10^{3}$ \\
& 100 & $7.5 \times 10^{-2}$ & -62.5 & $10^{4} \sim 10^{5}$ \\
& 180 & $1.5 \times 10^{-2}$ & -37.0 & $10^{4} \sim 10^{5}$ \\
\hline
\end{tabular}

发生变化, 进而影响了 OFET 器件的阈值电压.

\section{4 薄膜形貌表征}

为了研究材料的薄膜形貌和器件性能之间的关系, 采用 X 射线衍射(XRD)和原子力显微镜(AFM)对材料的 薄膜形貌进行表征. 从 XRD 曲线(图 5)可以看出, 对于 未退火的薄膜, 光学纯 $(\mathrm{I}-\boldsymbol{R}, \mathrm{I}-\mathrm{S})$ 与消旋体材料 $(\mathrm{I}-\boldsymbol{R}, \boldsymbol{S}$, I-rac)均出现两组一致的一级衍射峰, $2 \theta$ 分别约为 $5.25^{\circ}$ 和 $6.25^{\circ}$, 相应的 $d$-spacing 距离分别为 1.68 和 $1.41 \mathrm{~nm}$, 表明未退火薄膜中可能存在两种结晶相. 薄膜经 $100{ }^{\circ} \mathrm{C}$ 热退火后, 图谱中仅出现一组增强的一级衍射峰, $2 \theta$ 约 为 $5.28^{\circ}$, 相应的 $d$-spacing 距离分别为 $1.67 \mathrm{~nm}$. 这表明 材料的晶相发生转变形成更有序的结晶, 同时出现微弱 的多级衍射峰, 其 $2 \theta$ 约为 $16.0^{\circ}$, 相应的 $d$-spacing 距离 分别为 $0.55 \mathrm{~nm}$, 说明分子排列具有一定的长程有序性.
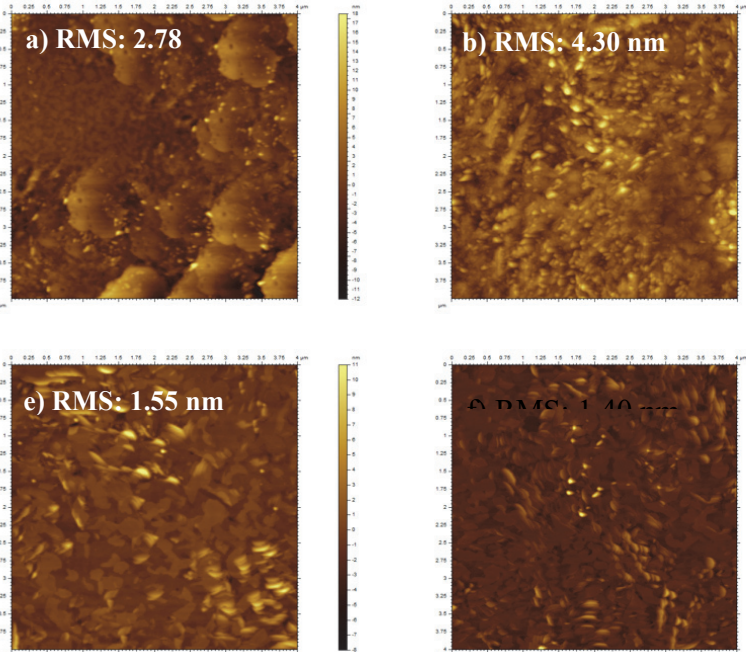
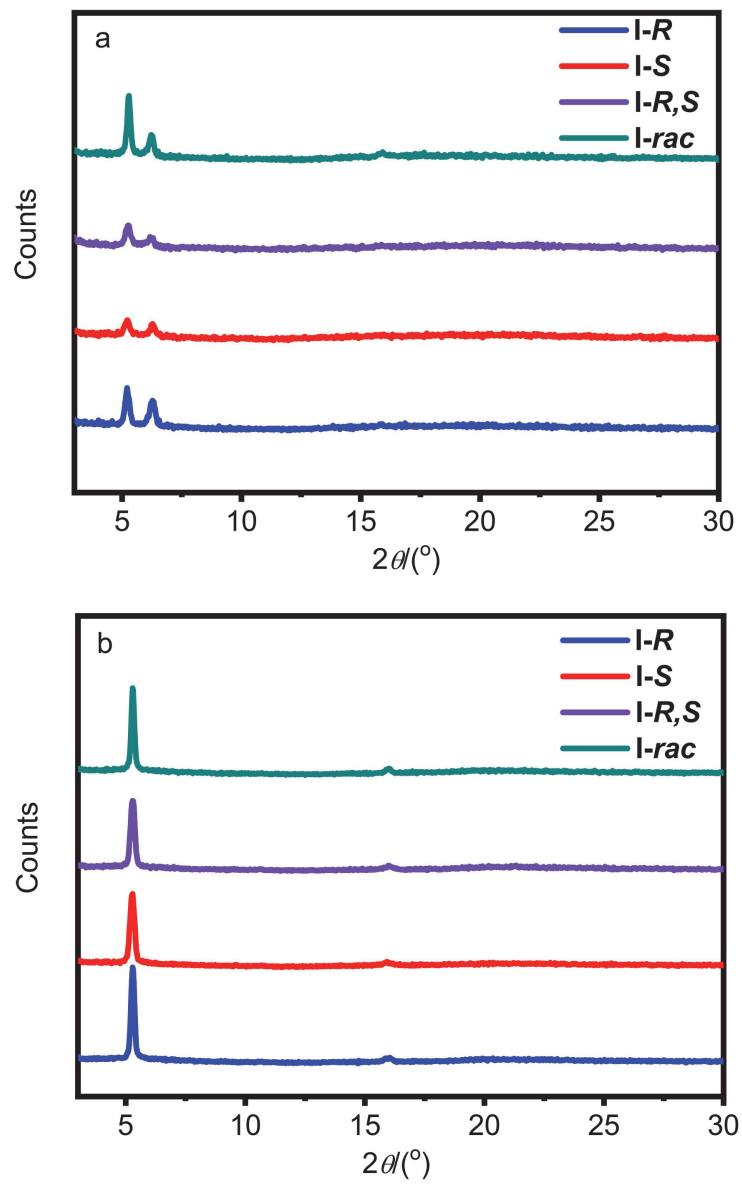

图 $5 \mathrm{I}-\boldsymbol{R}, \mathrm{I}-\mathrm{S}, \mathrm{I}-\boldsymbol{R}, \boldsymbol{S}$ 和 I-rac 的薄膜的 XRD 衍射图谱 Figure 5 XRD patterns of the films of I-R, I-S, I-R,S and I-rac (a) as-spun, (b) annealed at $100{ }^{\circ} \mathrm{C}$

图 6 显示了四种材料薄膜在未退火 $(\mathrm{a}, \mathrm{b}, \mathrm{c}, \mathrm{d})$ 和 $100{ }^{\circ} \mathrm{C}$ 热 退火(e, f, g, h) 条件下的 AFM 高度图. 对于光学纯材料 $\mathbf{I}-\boldsymbol{R}$ 和 $\mathbf{I}-\boldsymbol{S}, 100{ }^{\circ} \mathrm{C}$ 热退火后，由于材料薄膜结晶性增强,
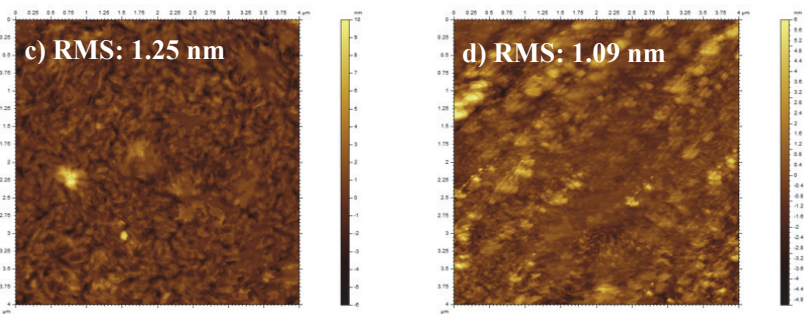

图 $6 \mathbf{I}-\boldsymbol{R}(\mathrm{a}, \mathrm{e}), \mathbf{I}-\boldsymbol{S}(\mathrm{b}, \mathrm{f}), \mathbf{I}-\boldsymbol{R}, \boldsymbol{S}(\mathrm{c}, \mathrm{g})$ 和 I-rac (d, h) 薄膜在未退火 $(\mathrm{a}, \mathrm{b}, \mathrm{c}, \mathrm{d})$ 和 $100{ }^{\circ} \mathrm{C}$ 热退火 $(\mathrm{e}, \mathrm{f}, \mathrm{g}, \mathrm{h})$ 条件下的 $\mathrm{AFM}$ 图 Figure 6 AFM height images of as-spun thin films of (a) I-R, (b) I-S, (c) I-R,S and (d) I-rac, and AFM height images of thin films of (e) $\mathbf{I}-\boldsymbol{R}$, (f) $\mathbf{I}-\boldsymbol{S}$, (g) $\mathbf{I}-\boldsymbol{R}, \boldsymbol{S}$ and (h) I-rac annealed at $100{ }^{\circ} \mathrm{C}$ 
退火薄膜较未退火薄膜显示出更均匀的形貌以及更小 的均方根(root mean square, RMS)粗楉度 (100 ${ }^{\circ} \mathrm{C}$ 热退火 后, I- $\boldsymbol{R}$ 和 I-S 薄膜的 RMS 分别为 $1.55 \mathrm{~nm}$ 和 $1.40 \mathrm{~nm}$ ), 但存在较小的晶粒, 这意味着更多的晶界不利于载流子 的传输. 而消旋体材料 I-R, S 和 I-rac 的薄膜在 $100{ }^{\circ} \mathrm{C}$ 热 退火后, 由于结晶度的提高及长程有序性的增加, 薄膜 的晶粒尺寸和粗粘度因更强的聚集和成核作用而有所 增大, 并且晶界明显减少. 因此, 薄膜经 $100{ }^{\circ} \mathrm{C}$ 热退火 后, 消旋体 $(\mathbf{I}-\boldsymbol{R}, \boldsymbol{S}, \mathbf{I}-\boldsymbol{r a c})$ 的 OFET 器件性能优于光学纯 材料(I-R, I-S).

\section{2 结论}

用四溴荎二酰亚胺和手性环己二胺经亲核取代反 应合成了三个萗二酰亚胺类小分子 I- $R, \mathrm{I}-\mathrm{S}$ 和 I- $R, S$, 将 等量的 I-R 和 I-S 溶于二氯甲烷, 经甲醇沉析得到了外 消旋体 I-rac, 并用 TGA, DSC, CV 和紫外-可见吸收光 谱等手段研究了四种材料的物理化学性质. 四种材料在 二氯甲烷溶液中的光谱吸收完全一致, 但薄膜状态下的 吸收有所差异. 消旋体(I-R,S, I-rac) 薄膜的最大吸收峰 较光学纯化合物(I-R, I-S)红移了约 $5 \mathrm{~nm}$, 表明它们的聚 集态结构存在一定差别. 此外, 还对材料的薄膜 OFET 性能进行了研究. 在 $100{ }^{\circ} \mathrm{C}$ 热退火条件下, 内消旋体 $(\mathbf{I}-\boldsymbol{R}, \boldsymbol{S})$ 和外消旋体(I-rac) OFET 器件的空穴迁移率分别 为 0.074 和 $0.075 \mathrm{~cm}^{2} \cdot \mathrm{V}^{-1} \cdot \mathrm{s}^{-1}$, 均高于光学纯材料 $\mathbf{I}-\boldsymbol{R}$ 和 $\mathbf{I}-\boldsymbol{S}$ 的器件性能(空穴迁移率约为 $0.04 \mathrm{~cm}^{2} \cdot \mathrm{V}^{-1} \cdot \mathrm{s}^{-1}$ ). 薄 膜经 $180{ }^{\circ} \mathrm{C}$ 热退火处理后, 光学纯材料 $\mathbf{I}-\boldsymbol{R}$ 和 $\mathbf{I}-\boldsymbol{S}$ 的空 穴迁移率表现出数量级的下降, 消旋体材料 I-R,S 和 I-rac 仅略有下降. 薄膜形貌研究表明, 消旋体材料的聚 集态结构更有利于电荷传输.

\section{3 实验部分}

\section{1 仪器与试剂}

核磁共振 $\left({ }^{1} \mathrm{H}\right.$ NMR, ${ }^{13} \mathrm{C}$ NMR $)$ 由 JEOL 400 核磁共 振波谱仪测定, 采用 $\mathrm{CDCl}_{3}$ 作为溶剂, $\mathrm{TMS}$ 作为内标; IR 由 Thermo Fisher Nicolet FT-IR spectrometer 型红外分析 仪测定; 质谱(MS) MALDI-TOF 由 Bruker Biflex III MDLDI-TOF 质谱仪测定; HRMS (MALDI)由 Thermo Fisher Scientific LTQ FT Ultra 质谱仪测定; 元素分析由 Elementar Vario EL III型元素分析仪测定; 熔点由上海 申光 SGWX-4 显微熔点仪测定; 热重分析(TGA)由 TGA Q500 型热重分析仪测定, 测试条件为氮气氛围, 升温 速度为 $10{ }^{\circ} \mathrm{C} / \mathrm{min}$; 差热分析(DSC)由 DSC Q2000 仪器 测定, 测试条件为氮气氛围, 升/降温速度为 $10{ }^{\circ} \mathrm{C} / \mathrm{min}$. 紫外-可见吸收光谱(UV-vis)由 U-3900 光谱仪测得; 电 子圆二色谱 $(E C D)$ 在 Chirascan instrument 光谱仪上测得;
电化学测试(CV)在计算机控制的 CHI610D 电化学分析 仪上进行, 采用传统的三电极测试方法, 饱和甘录电极 作为参比电极、玻碳电极作为工作电极、铂丝作为对电 极, $\mathrm{Bu}_{4} \mathrm{NPF}_{6}(0.1 \mathrm{~mol} / \mathrm{L})$ 作为支持电解质, 将样品溶于二 氯甲烷溶液中进行测试, 扫描速率为 $100 \mathrm{mV} / \mathrm{s}$; OFET 器件采用底柾极顶接触(BGTC) 结构, 通过旋涂的方法 将样品沉积在十八烷基三氯硅烷(OTs)修饰的 $\mathrm{Si} / \mathrm{SiO}_{2}$ 基 底上，在不同的温度下进行退火处理来改善薄膜的结构 形貌，利用掩模板沉积金电极，厚度为 $50 \mathrm{~nm}$, 器件沟 道长度为 $31 \mu \mathrm{m}$, 沟道宽度为 $273 \mu \mathrm{m}$; 器件性能由 Keithley 4200 半导体测试仪在室温氮气氛围中测得; 原 子力显微镜(AFM)图由 JPK NanoWizard Sense 原子力显 微镜测得, 测试模式为 tapping 模式; $X$ 射线衍射 $(X R D)$ 由 PANalytical X'Pert PRO 系统测定.

\section{2 实验方法}

\subsubsection{2,3,6,7-四溴-1,4,5,8-菜二酸酐(I-2)的合成}

参考文献[21]. 向干燥的 $350 \mathrm{~mL}$ 封管中加入 1,4,5,8-菜二酸酐 I-1 (10 g, $37.0 \mathrm{mmol}, 1.0$ equiv.), 浓硫 酸 $(75 \mathrm{~mL})$, 摚拌溶解后, 分批次加入二溴海因 (DBH) (37.3 g, $131.0 \mathrm{mmol}, 3.5$ equiv.), 加热至 $60{ }^{\circ} \mathrm{C}$ 反应 $96 \mathrm{~h}$. 反应结束后, 冷却至室温, 缓慢打开瓶塞, 将反应液倾 倒入冰水中, 静置 $0.5 \mathrm{~h}$, 减压抽滤, 乙醇洗涤滤饼直至 滤液呈无色，滤饼真空干燥过夜，得金黄色固体 I-2 (13 g), 收率 $60 \%$, m.p. $>350{ }^{\circ} \mathrm{C}^{[24,25]}$, 无需纯化直接用于下 一步反应. 注意: 减压抽滤时, 抽滤瓶中加入一定浓度 的硫代硫酸钠以除去反应产生的 $\mathrm{Br}_{2}$, 以防其溢出.

\subsection{2 $N, N^{\prime}$-二正辛基-2,3,6,7-四溴-1,4,5,8-菜二酰亚} 胺(I-3)的合成

参考文献[22]. 向 $150 \mathrm{~mL}$ 单口瓶中加入 $\mathbf{I}-2(1 \mathrm{~g}$, $1.71 \mathrm{mmol}, 1.0$ equiv. $)$ 二氯甲烷 $(20 \mathrm{~mL})$, 搅拌溶解后 加入正辛胺 $\left(0.6 \mathrm{~mL}, 3.60 \mathrm{mmol}, 2.1\right.$ equiv.), 于 $40{ }^{\circ} \mathrm{C}$ 反 应 $8 \mathrm{~h}$. 反应结束后, 加入三溴化磷 $(1 \mathrm{~mL})$, 回流 $1 \mathrm{~h}$ 后 停止加热, 冷却至室温. 加水淬灭反应, 用二氯甲烷萃 取合并有机相，有机相用无水硫酸钠干燥，减压浓缩得 橙色粗品. 丙酮洗涤，过滤得黄色固体 I-3 ${ }^{[26]}$ (630 mg), 收率 46\%. ${ }^{1} \mathrm{H}$ NMR (400 MHz, $\left.\mathrm{CDCl}_{3}\right) \delta: 4.20$ (t, $J=8.0$ $\mathrm{Hz}, 4 \mathrm{H}), 1.75(\mathrm{~m}, 4 \mathrm{H}), 1.47 \sim 1.20(\mathrm{~m}, 20 \mathrm{H}), 0.88$ (t, $J=$ $8.0 \mathrm{~Hz}, 6 \mathrm{H})$

$3.2 .3(8 R, 13 R)$-八氢喹喔啉并 $N, N^{\prime}$-二正辛基-2,3-二 溴菜二酰亚胺(I-4)的合成

向 $50 \mathrm{~mL}$ 单口瓶中加入 $\mathbf{I - 3}(200 \mathrm{mg}, 0.248 \mathrm{mmol}$, 1.0 equiv.), 甲苯 $(10 \mathrm{~mL})$, 搅拌溶解后加入 $(1 R, 2 R)-(-)$ 1,2-环已二胺 $(28.33 \mathrm{mg}, 0.248 \mathrm{mmol}, 1.0$ equiv.), 于 $50{ }^{\circ} \mathrm{C}$ 下过夜反应. 反应结束后, 旋干反应液, 柱层析分 离纯化(柱层析采用中性氧化铝, 洗脱剂为 $[V$ (石油醚)： 
$V($ 二氯甲烷 $)=1: 1]$ 得橙色固体 $\mathbf{I}-\mathbf{4}(188 \mathrm{mg})$, 收率 $78 \%$. m.p. $228 \sim 230{ }^{\circ} \mathrm{C}$; ${ }^{1} \mathrm{H}$ NMR $\left(400 \mathrm{MHz}, \mathrm{CDCl}_{3}\right) \delta$ : 10.89 (s, 2H), $4.15 \sim 4.07(\mathrm{~m}, 4 \mathrm{H}), 3.31(\mathrm{~s}, 2 \mathrm{H}), 2.24(\mathrm{~s}$, $2 \mathrm{H}), 1.97(\mathrm{~d}, J=8.0 \mathrm{~Hz}, 2 \mathrm{H}), 1.69(\mathrm{~m}, 4 \mathrm{H}), 1.55(\mathrm{~s}, 4 \mathrm{H})$, $1.42 \sim 1.27(\mathrm{~m}, 20 \mathrm{H}), 0.88(\mathrm{t}, J=6.0 \mathrm{~Hz}, 6 \mathrm{H}) ;{ }^{13} \mathrm{C} \mathrm{NMR}$ $\left(100 \mathrm{MHz}, \mathrm{CDCl}_{3}\right) \delta: 165.29,160.89,144.53,126.61$, $124.17,121.72,96.86,54.12,41.76,32.28,30.68,29.75$, 28.33, 27.76, 24.41, 23.11, 14.56; FT-IR (KBr) v: 2916.83, 2848.48, 1678.32, 1630.23, 1591.12, 1567.84, 1531.95, $1456.21,1346.19,1296.47,1259.63,1243.54,1233.92$, $786.73,720.58,702.72,661.90 \mathrm{~cm}^{-1}$; HRMS (MALDITOF) calcd for $\mathrm{C}_{36} \mathrm{H}_{47} \mathrm{~N}_{4} \mathrm{O}_{4} \mathrm{Br}_{2}[\mathrm{M}+\mathrm{H}]^{+}$757.19917; found 757.19586. Anal. calcd for $\mathrm{C}_{36} \mathrm{H}_{46} \mathrm{~N}_{4} \mathrm{O}_{4} \mathrm{Br}_{2}$ : C 57.00, $\mathrm{H}$ 6.11, N 7.39; found C 57.03, H 6.13, N 7.30.

3.2.4 $(4 R, 9 R, 16 R, 21 R)-N, N^{\prime}$-二正辛基-双八氢喹喔啉 并- $1,4,5,8$ - 菜二酰亚胺 $(\mathbf{I}-\boldsymbol{R})$ 的合成

向 $50 \mathrm{~mL}$ 单口瓶中加入 $\mathbf{I}-3(600 \mathrm{mg}, 0.74 \mathrm{mmol}, 1.0$ equiv.), 甲苯 $(10 \mathrm{~mL})$, 搅拌溶解后加入 $(1 R, 2 R)-(-)-1,2-$ 环已二胺 (255 mg, $2.23 \mathrm{mmol}, 3.0$ equiv.), 于 $110{ }^{\circ} \mathrm{C}$ 下回 流反应 $5 \mathrm{~h}$. 反应结束后, 旋干反应液, 柱层析分离纯化 [柱层析采用中性氧化铝, 洗脱剂为 $V$ (石油醚) : $V$ (二氯 甲烷 $)=3: 1]$, 得蓝紫色固体 I-R $(340 \mathrm{mg})$, 收率 $64 \%$. m.p. $270 \sim 272{ }^{\circ} \mathrm{C} ;{ }^{1} \mathrm{H}$ NMR (400 MHz, $\mathrm{CDCl}_{3}$ ) $\delta$ : 9.64 (s, $4 \mathrm{H}), 4.06(\mathrm{dd}, J=8.0,8.0 \mathrm{~Hz}, 4 \mathrm{H}), 3.09(\mathrm{~s}, 4 \mathrm{H}), 2.09$ (d, $J=8.0 \mathrm{~Hz}, 4 \mathrm{H}), 1.89$ (d, $J=8.0 \mathrm{~Hz}, 4 \mathrm{H}), 1.63(\mathrm{~m}, 4 \mathrm{H})$, $1.44 \sim 1.24(\mathrm{~m}, 28 \mathrm{H}), 0.87(\mathrm{t}, J=8.0 \mathrm{~Hz}, 6 \mathrm{H}) ;{ }^{13} \mathrm{C} \mathrm{NMR}$ $\left(100 \mathrm{MHz}, \mathrm{CDCl}_{3}\right) \delta: 166.35,139.40,113.28,100.27$, $53.88,40.75,32.33,31.03,29.96,29.77,28.65,27.88$, 24.68, 23.11, 14.57; FT-IR (KBr) v: 3241.09, 2921.77, $2851.10,1646.21,1617.03,1578.88,1508.34,1462.07$, $1336.76,1280.62,1260.36,1238.15,1227.02,1176.63$, $1152.19,1117.68,790.92,668.42 \mathrm{~cm}^{-1}$; HRMS (MALDITOF) calcd for $\mathrm{C}_{42} \mathrm{H}_{58} \mathrm{~N}_{6} \mathrm{O}_{4} 710.45141$; found 710.45347 . Anal. calcd for $\mathrm{C}_{42} \mathrm{H}_{58} \mathrm{~N}_{6} \mathrm{O}_{4}$ : C 70.95, H 8.22, N 11.82; found $\mathrm{C} 71.18, \mathrm{H}$ 8.26, N 11.76 .

$3.2 .5(4 S, 9 S, 16 S, 21 S)-N, N^{\prime}$-二正辛基-双八氢喹喔啉 并-1,4,5,8-蔡二酰亚胺 $(\mathbf{I}-\boldsymbol{S})$ 的合成

向 $50 \mathrm{~mL}$ 单口瓶中加入 $\mathbf{I}-\mathbf{3}(600 \mathrm{mg}, 0.74 \mathrm{mmol}, 1.0$ equiv.), 甲苯 $(10 \mathrm{~mL})$, 搅拌溶解后加入 $(1 S, 2 S)-(+)-1,2-$ 环已二胺( $255 \mathrm{mg}, 2.23 \mathrm{mmol}, 3.0$ equiv.), 于 $110{ }^{\circ} \mathrm{C}$ 下回 流反应 $5 \mathrm{~h}$. 反应结束后, 旋干反应液, 柱层析分离纯化 (柱层析采用中性氧化铝, 洗脱剂为 $[V$ (石油醚) $: V$ (二氯 甲烷 $)=3: 1$ ], 得蓝紫色固体 I-S $(295 \mathrm{mg})$, 收率 $56 \%$. m.p. $268 \sim 270{ }^{\circ} \mathrm{C} ;{ }^{1} \mathrm{H}$ NMR $\left(400 \mathrm{MHz}, \mathrm{CDCl}_{3}\right) \delta$ : 9.62 (s, $4 \mathrm{H}), 4.07$ (dd, $J=8.0,8.0 \mathrm{~Hz}, 4 \mathrm{H}), 3.13(\mathrm{~s}, 4 \mathrm{H}), 2.10$ (d,
$J=8.0 \mathrm{~Hz}, 4 \mathrm{H}), 1.90(\mathrm{~d}, J=8.0 \mathrm{~Hz}, 4 \mathrm{H}), 1.64(\mathrm{~m}, 4 \mathrm{H})$, $1.43 \sim 1.25(\mathrm{~m}, 28 \mathrm{H}), 0.88(\mathrm{t}, J=8.0 \mathrm{~Hz}, 6 \mathrm{H}) ;{ }^{13} \mathrm{C}$ NMR $\left(100 \mathrm{MHz}, \mathrm{CDCl}_{3}\right) \delta: 166.26,139.31,113.15,100.05$, $53.76,40.76,32.33,31.05,29.95,29.77,28.64,27.89$, 24.69, 23.10, 14.56; FT-IR (KBr) v: 3241.09, 2921.77, $2851.10,1646.21,1617.03,1578.88,1508.34,1462.07$, 1336.76, 1280.62, 1260.36, 1238.15, 1227.02, 1176.63, $1152.19,1117.68,790.92,668.42 \mathrm{~cm}^{-1}$; HRMS (MALDITOF) calcd for $\mathrm{C}_{42} \mathrm{H}_{58} \mathrm{~N}_{6} \mathrm{O}_{4} 710.45141$; found 710.45347 . Anal. calcd for $\mathrm{C}_{42} \mathrm{H}_{58} \mathrm{~N}_{6} \mathrm{O}_{4}$ : C 70.95, H 8.22, N 11.82; found $\mathrm{C} 71.10, \mathrm{H} 8.40, \mathrm{~N} 11.94$.

3.2.6 (4R, $9 R, 16 S, 21 S)-N, N^{\prime}$-二正辛基-双八氢喹喔啉 并-1,4,5,8-菜二酰亚胺 $(\mathbf{I}-\boldsymbol{R}, \boldsymbol{S})$ 的合成

向 $50 \mathrm{~mL}$ 单口瓶中加入 $\mathbf{I}-4(300 \mathrm{mg}, 0.395 \mathrm{mmol}$, 1.0 equiv.), 甲苯 $(10 \mathrm{~mL})$, 搅拌溶解后加入 $(1 S, 2 S)-(+)-$ 1,2-环己二胺 $(67.7 \mathrm{mg}, 0.593 \mathrm{mmol}, 1.5$ equiv.), 于 $110{ }^{\circ} \mathrm{C}$ 下回流反应 $5 \mathrm{~h}$. 反应结束后, 旋干反应液, 柱层 析分离纯化 [柱层析采用中性氧化铝, 洗脱剂为 $V$ (石油 醚) : $V$ (二氯甲烷 $)=3: 1]$, 得蓝紫色固体 I-R,S (190 $\mathrm{mg}$ ), 收率 68\%. m.p. 272.9 274.5 ${ }^{\circ} \mathrm{C} ;{ }^{1} \mathrm{H}$ NMR (400 $\left.\mathrm{MHz}, \mathrm{CDCl}_{3}\right) \delta: 9.74(\mathrm{~s}, 4 \mathrm{H}), 4.12(\mathrm{~s}, 4 \mathrm{H}), 3.15(\mathrm{~s}, 4 \mathrm{H})$, $2.14(\mathrm{~s}, 4 \mathrm{H}), 1.89(\mathrm{~s}, 4 \mathrm{H}), 1.68(\mathrm{~s}, 4 \mathrm{H}), 1.44 \sim 1.28(\mathrm{~m}$, $28 \mathrm{H}), 0.88(\mathrm{~s}, 6 \mathrm{H}) ;{ }^{13} \mathrm{C}$ NMR $\left(100 \mathrm{MHz}, \mathrm{CDCl}_{3}\right) \delta$ : 166.36, 139.44, 113.38, 100.56, 53.72, 40.75, 32.33, 31.06, 29.96, 29.78, 28.78, 27.89, 24.69, 23.11, 14.57; FT-IR (KBr) v: 3243.70, 2922.07, 2851.10, 1644.51, 1614.89, $1577.21,1506.78,1462.95,1334.89,1284.80,1268.36$, $1237.90,1219.33,1176.26,1153.89,1114.95,790.55$ $\mathrm{cm}^{-1}$; HRMS (MALDI-TOF) calcd for $\mathrm{C}_{42} \mathrm{H}_{58} \mathrm{~N}_{6} \mathrm{O}_{4}$ 710.45141; found 710.44996. Anal. calcd for $\mathrm{C}_{42} \mathrm{H}_{58} \mathrm{~N}_{6} \mathrm{O}_{4}$ : C 70.95, H 8.22, N 11.82; found C 71.00, H 8.32, N 11.67.

辅助材料(Supporting Information) 热重分析曲线、差 示扫描量热曲线、单晶结构、 $\mathrm{X}$ 射线衍射图、原子力显 微镜高度图、核磁谱图、高分辨质谱图和红外光谱. 这 些材料可以免费从本刊网站(http://sioc-journal.cn/)上下 载.

\section{References}

[1] Kuang, H.; Xu, C.; Tang, Z. Adv. Mater. 2020, 32, 2005110.

[2] Liu, M. Acta Phys. Chim. Sin. 2020, 36, 2004031 (in Chinese). (刘鸣华, 物理化学学报, 2020, 36, 2004031.)

[3] Liu, J.; Yin, F.; Hu, J.; Ju, Y. Chin. J. Org. Chem. 2021, 41, 1031 (in Chinese).

(刘金果, 殷凤, 胡君, 巨勇, 有机化学, 2021, 41, 1031.)

[4] Pasteur, L. Ann. Chim. Phys. 1848, 24, 442.

[5] Reinitzer, F. Monatsh. Chem. 1888, 9, 421.

[6] Geng, Y.; Trajkovska, A.; Culligan, S. W.; Ou, J. J.; Chen, H. M. P.; 
Katsis, D.; Chen, S. H. J. Am. Chem. Soc. 2003, 125, 14032.

[7] Yang, Y.; da Costa, R. C.; Smilgies, D. M.; Campbell, A. J.; Fuchter, M. J. Adv. Mater. 2013, 25, 2624.

[8] Liu, J.; Su, H.; Meng, L.; Zhao, Y.; Deng, C.; Ng, J. C. Y.; Lu, P.; Faisal, M.; Lam, J. W. Y.; Huang, X.; Wu, H.; Wong, K. S.; Tang, B. Chem. Sci. 2012, 3, 2737.

[9] Hatakeyama, T.; Hashimoto, S.; Oba, T.; Nakamura, M. J. Am. Chem. Soc. 2012, 134, 19600.

[10] Liu, J.; Zhang, Y.; Phan, H.; Sharenko, A.; Moonsin, P.; Walker, B.; Promarak, V.; Nguyen, T. Q. Adv. Mater. 2013, 25, 3645.

[11] Ying, Y.; Rice, B.; Shi, X.; Brandt, J. R.; Correa da Costa, R.; Hedley, G. J.; Smilgies, D. M.; Frost, J. M.; Samuel, I. D. W.; Otero-de-la-Roza, A.; Johnson, E. R.; Jelfs, K. E.; Nelson, J.; Campbell, A. J.; Fuchter, M. J. ACS Nano 2017, 11, 8329.

[12] Chen, M.; Jiao, X. C.; Li, J.; Wu, W.; Xin, H.; McNeill, C. R.; Gao, $\mathrm{X}$. Langmuir 2019, 35, 6188.

[13] Sakai, N.; Mareda, J.; Vauthey, E.; Matile. Chem. Commun. 2010, $46,4225$.

[14] Bhosale, S. V.; Bhosale, S. V.; Bhargava, S. K. Org. Biomol. Chem. 2012, 10, 6455 .

[15] Suraru, S. L.; Würthner, F. Angew. Chem., Int. Ed. 2014, 53, 7428.

[16] Guo, D.; Li, L.; Zhu, X.; Heeney, M.; Li, J.; Dong, L.; Yu, Q.; Gan,
Z.; Gu, X.; Tan, L. Sci. China Chem. 2020, 63, 1198.

[17] Zhang, F.; Hu, Y.; Schuettfort, T.; Di, C.; Gao, X.; McNeill, C. R.; Thomsen, L.; Mannsfeld, S. C. B.; Yuan, W.; Sirringhaus, H.; Zhu, D. J. Am. Chem. Soc. 2013, 135, 2338.

[18] Zhao, Z.; Zhang, F. J.; Hu, Y.; Wang, Z.; Leng, B.; Gao, X.; Di, C.; Zhu, D. ACS Macro Lett. 2014, 3, 1174.

[19] Wu, W.; Zhao, Z.; Li, J.; Chen, M.; Gao, X. Asian J. Org. Chem. 2018, 7, 2279.

[20] Han, W.; Wang, Z.; Hu, Y.; Yang, X.; Ge, C.; Gao, X. Sci. China Chem. 2020, 63, 1182.

[21] Sasikumar, M.; Suseela, Y. V.; Govindaraju, T. Asian J. Org. Chem. 2013, 2, 779 .

[22] Hu, Y.; Wang, Z.; Yang, X.; Zhao, Z.; Han, W.; Yuan, W.; Li, H.; Gao, X.; Zhu, D. Tetrahedron Lett. 2013, 54, 2271.

[23] Jelley, E. E. Nature 1936, 138, 1009.

[24] Gao, X.; Qiu, W.; Yang, X.; Liu, Y.; Wang, Y.; Zhang, H.; Qi, T.; Liu, Y.; Lu, K.; Du, C.; Shuai, Z.; Yu, G.; Zhu, D. Org. Lett. 2007, 9, 3917

[25] Röger, C.; Würthner, F. J. Org. Chem. 2007, 72, 8070.

[26] Gao, X.; Qiu, W.; Yang, X.; Liu, Y.; Wang, Y.; Zhang, H.; Qi, H.; Liu, Y.; Lu, K.; Du, C.; Shuai, Z.; Yu, G.; Zhu, D. Org. Lett. 2007, 9, 3917

(Li, L.; Fan, Y.) 